\section{Laws of physics help explain capillary non- perfusion in diabetic retinopathy}

\begin{abstract}
The purpose is to use laws of physics to elucidate the mechanisms behind capillary non-perfusion in diabetic retinopathy. In diabetic retinopathy, loss of pericytes weakens capillary walls and the vessel dilates. A dilated capillary has reduced resistance to flow, therefore increased flow in that vessel and decreased in adjoining capillaries. A preferential shunt vessel is thus formed from the dilated capillary and the adjacent capillaries become non-perfused. We apply the laws of Laplace and HagenPoiseuille to better understand the phenomena that lead to capillary nonperfusion. These laws of physics can give a foundation for physical or mathematical models to further elucidate this field of study. The law of Laplace predicts that a weaker vessel wall will dilate, assuming constant transmural pressure. The Hagen-Poiseuille equation for flow and the Ostwald-de Waele relationship for viscosity predict that a dilated vessel will receive a higher portion of the fluid flow than the adjoining capillaries. Viscosity will decrease in the dilated vessel, furthering the imbalance and resulting in a patch of non-perfused capillaries next to the dilated 'preferential' shunt vessel. Physical principles support or inspire novel hypotheses to explain poorly understood phenomena in ophthalmology. This thesis of pericyte death and capillary remodelling, which was first proposed by Cogan and Kuwabara, already agrees with histological and angiographical observations in diabetic retinopathy. We have shown that it is also supported by classical laws of physics. Eye (2018) 32, 210-212; doi:10.1038/eye.2017.313; published online 19 January 2018
\end{abstract}

The pathophysiology of diabetic retinopathy and the vascular changes have been investigated using several different technological approaches,
E Stefánsson 1, YK Chan², T Bek³ ${ }^{3}$ SH Hardarson ${ }^{4}$, D Wong ${ }^{5}$ and DI Wilson ${ }^{6}$ but we are still far away from a comprehensive understanding of this process. One of the seemingly paradoxical observations is the occurrence of capillary non-perfusion with hyperperfused shunt vessels and adjacent capillary occlusion in the same retinal area (Figure 1). This might at first glance suggest the presence of opposite causal factors in the disease process.

The histological studies of Cogan and Kuwabara discovered some of the early changes in diabetic retinopathy. ${ }^{1,2}$ They demonstrated death of pericytes in the retinal capillaries and the appearance of non-perfused 'ghost' vessels in the capillary bed. They described 'preferential channels' which are shunt vessels (Figure 2).

These preferential channels are dilated capillaries that transcend or are adjacent to patches of non-perfused capillaries in the retina.

The histological picture points directly to the pathophysiological mechanism. Pericytes die due to hyperglycaemia. Pericytes (mural cells) are contractile cells and strengthen the capillary wall. ${ }^{3}$ When they perish, according to the law of Laplace, the wall strength is reduced and the vessel dilates due to the intravascular-tissue pressure difference. All of this was elegantly stated by Cogan and Kuwabara: ${ }^{1}$ 'The pathogenesis of diabetic retinopathy thus revolves about a loss of tone in the capillaries, and this loss permits flow through certain channels - a process that is characteristic of diabetes. It has been previously suggested that the mural cells are responsible for the tonic control of the retinal capillaries. The mural cells presumably guarantee the uniform distribution of blood through all normal capillaries of the elaborate plexus fed by single arterioles. With diabetes these mural cells characteristically disappear'. ......'In diabetes circulation continues through the endothelial lined capillaries but flow is no longer under the tonic control of mural cells and shunts develop. Although these shunt vessels arise from preformed capillaries, they are 


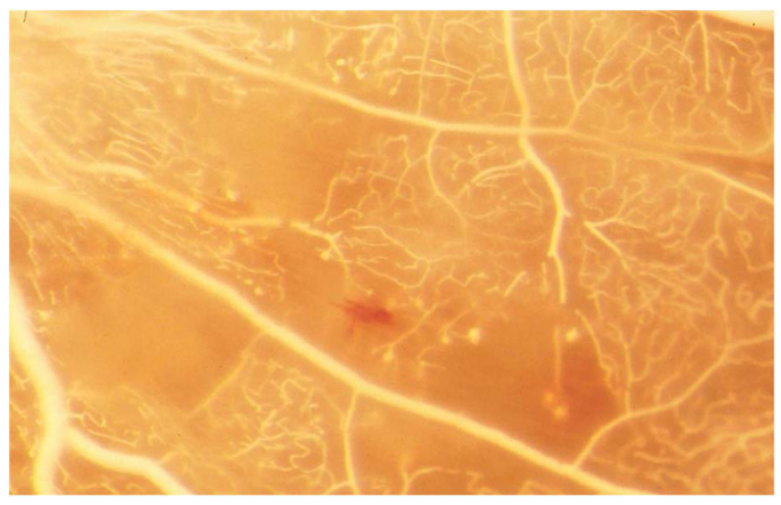

Figure 1 Human retina with a cast of the vascular tree post mortem. Microaneurysms are seen to bound areas of capillary occlusion traversed by larger patent vessels. The red dot represents a small haemorrhage in the nerve fibre layer.

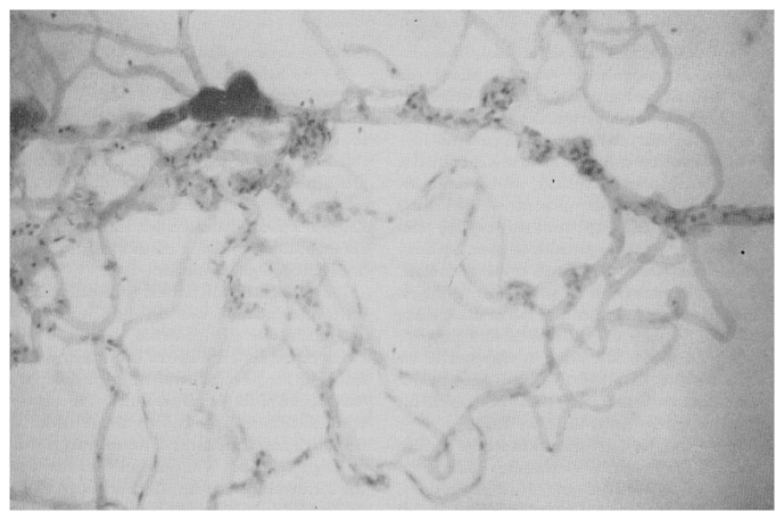

Figure 2 Shunt capillaries with microaneurysms from the retina of a diabetic patient with moderate retinopathy. ${ }^{1}$ Note that the shunt vessel has an increased cellularity while the adjacent occluded capillaries are acellular. $80 \times$. Reproduced with permission from the American Diabetes Association.

often interpreted clinically as neovascularization.' ......

'The relationship of these shunts to the acellular capillaries which have been bypassed shows clearly in flat mounts so long as the plexus of vessels is not too dense.'

The purpose of the current article is to use principles of physics to further examine the hypothesis by Cogan and Kuwabara.

\section{Physics}

In a capillary net, one capillary has suffered loss of pericytes (mural cells), its wall has weakened and dilates according to the law of Laplace. The dilated capillary has a larger diameter, $d$, and therefore a lower resistance to flow (Figure 3). This affects the local distribution of blood flow, which is best explained using the Hagen-Poiseuille equation for flow of a shear-thinning fluid along a tube of length $L$ which follows the Ostwald-de Waele relationship for viscosity: ${ }^{4}$
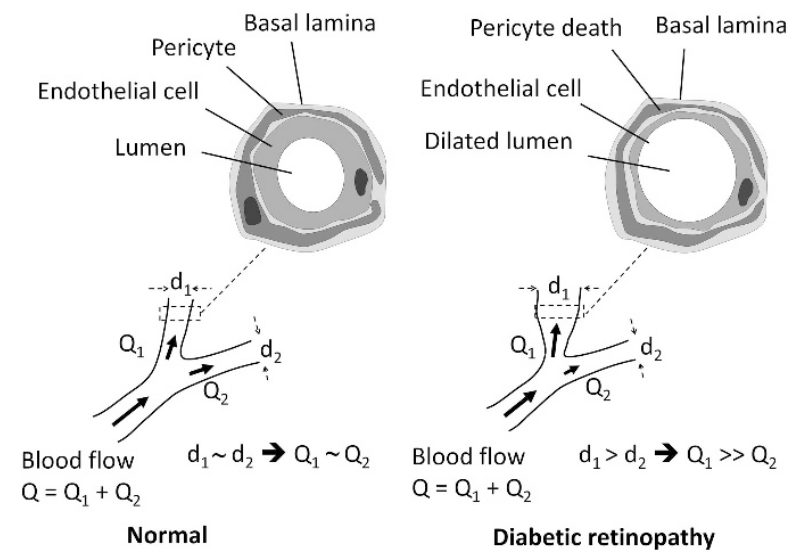

Figure 3 Schematic drawing where one capillary suffers pericyte death, weakening of the wall and subsequent dilatation. Blood flow is increased in the dilated vessels and reduced in the adjoining vessel. A vicious cycle leaves the vessel void of all blood flow as a non-perfused capillary. The dilated one becomes a preferential shunt vessel.

$Q=\frac{\pi d^{3}}{8\left(3+\frac{1}{n}\right)}\left(\frac{\Delta P d}{4 K L}\right)^{\frac{1}{n}}$

In this Equation $Q$ is the flow rate along a tube, $\Delta P$ the pressure difference between the artery and vein (effectively the same for capillaries near one another), $K$ is the viscosity parameter and $n$ is the power law index. Blood flow in capillaries is relatively slow so losses against viscous friction dominate. Blood is a shearthinning fluid: $5 n$ is $<1$ so the blood becomes more viscous as the flow slows down, and at low flow rates it can experience jamming where blood cells effectively get stuck $^{6}$ (this is not predicted by the Ostwald-de Waele relationship). Conversely, if $d$ increases for the same pressure drop the viscosity decreases and even more flows along the dilated vessel. If one sets $n=1 / 2$, the flow rate is proportional to $d^{5}$ : a $10 \%$ increase in $d$ gives a $60 \%$ increase in $Q$.

Dilation of one capillary will thus promote redistribution of flow between neighbouring capillaries. As the flow rate in a capillary drops the blood cells will tend to aggregate and jam. This is a vicious cycle that only stops when the surrounding capillaries have been rendered bloodless, that is, non-perfused, and turn into the ghost capillaries described by Cogan and Kuwabara ${ }^{1,2}$ (Figure 2).

This is an example of a manifold flow problem. It explains the co-existence of preferential shunt channels and capillary non-perfusion in the capillary bed in diabetic retinopathy. It explains why capillary nonperfusion occurs in relatively well-defined patches and at the same time why they are limited and do not engulf the entire retinal circulation. 


\section{Alternative hypotheses}

Other hypothesis on the development of capillary nonperfusion in diabetic retinopathy, have suggested an embolic mechanism. ${ }^{7}$ White blood cells have been pointed out at the culprit and proposed that they stick in some capillaries, occluding the vessel. ${ }^{8,9}$ If this were the case, we would expect the non-perfusion to be somewhat equally scattered at random over the entire capillary bed and in isolated capillaries as there would be no reason to expect adjacent vessels to be more susceptible than others. Indeed, the non-perfusion patches would not be expected if this was the mechanism nor does it provide any explanation for the preferential shunt vessels. Recently, Lechner et al ${ }^{10}$ simply stated that: 'Retinal capillaries become progressively non-perfused in the diabetic retina as a direct result vasodegeneration'. Histological studies have shown that this vascular occlusion can be related to basement membrane thickening ${ }^{11}$ and ingrowth of Müller cells from the surrounding perivascular retina. ${ }^{12,13}$ In addition, studies in experimental animals suggest the involvement of granulocyte plugs in the development of capillary occlusion ${ }^{7,8}$ but these observations are by lack of a good animal model for diabetic retinopathy. This is a cardinal feature of diabetic retinopathy as observed in post-mortem specimens but also in long-term diabetic animal models.

\section{Conclusion}

The laws of physics provide us with a foundation to understand nature and this includes the human animal and its diseases. Thorough study of classical physics will undoubtedly provide clearer and novel understanding of many eye diseases and their treatment. A theoretical approach is a prerequisite for sound hypotheses that can subsequently be put to scientific study and clinical trial. Lack of a theoretical approach has long been a weakness of ophthalmology, which is too data driven and all too willing to accept 'black box' explanations when it comes to pathophysiology or treatment mechanisms. We should learn from physics and other fields that have made great strides with a proper mixture of theory and practical study.

\section{Conflict of interest}

The authors declare no conflict of interest.

\section{References}

1 Cogan DG, Kuwabara T. Capillary shunts in the pathogenesis of diabetic retinopathy. Diabetes 1963; 12: 293-300.

2 Cogan DG, Toussaint D, Kuwabara T. Retinal vascular patterns. IV. Diabetic retinopathy. Arch Ophthalmol 1961; 66: 366-378.

3 Arboleda-Velasquez JF, Valdez CN, Marko CK, D'Amore PA. From pathobiology to the targeting of pericytes for the treatment of diabetic retinopathy. Curr Diab Rep 2015; 15(2): 573.

4 Power law (Ostwald-deWaele model). In: Gooch JW (ed.) Encyclopedic Dictionary of Polymers Springer: New York, NY, 2007 pp 781-781.

5 Anand M, Rajagopal KR. A shear-thinning viscoelastic fluid model for describing the flow of blood. Int J Cardiovasc Med Sci 2004; 4: 59-68.

6 Freund JB. The flow of red blood cells through a narrow spleen-like slit. Phys Fluids 2013; 25(11): 110807.

7 Hatchell DL, Wilson CA, Saloupis P. Neutrophils plug capillaries in acute experimental retinal ischemia. Microvasc Res 1994; 47(3): 344-354.

8 Schroder S, Palinski W, Schmid-Schonbein GW. Activated monocytes and granulocytes, capillary nonperfusion, and neovascularization in diabetic retinopathy. Am J Pathol 1991; 139(1): 81-100.

9 Kim SY, Johnson MA, McLeod DS, Alexander T, Hansen BC, Lutty GA. Neutrophils are associated with capillary closure in spontaneously diabetic monkey retinas. Diabetes 2005; 54(5): 1534-1542.

10 Lechner J, O'Leary OE, Stitt AW. The pathology associated with diabetic retinopathy. Vision Res 2017; 139: 7-14.

11 Ashton N. Vascular basement membrane changes in diabetic retinopathy. Montgomery lecture 1973. Br J Ophthalmol 1974; 58(4): 344-366.

12 Bek T. Glial cell involvement in vascular occlusion of diabetic retinopathy. Acta Ophthalmol Scand 1997; 75(3): 239-243.

13 Bek T. Immunohistochemical characterization of retinal glial cell changes in areas of vascular occlusion secondary to diabetic retinopathy. Acta Ophthalmol Scand 1997; 75(4): 388-392. 